\title{
Appendix: Executive summary of CUA guideline on adult overactive bladder
}

\author{
Jacques Corcos, MD, FRCSC'; Mikolaj Przydacz, MD'; Lysanne Campeau, MD, PhD, FRCSC'; Jonathan Witten, MD²; \\ Duane Hickling, MD, MSCl, FRCSC 3; Christiane Honeine, RN, BN'; Sidney B. Radomski, MD, FRCSC4; \\ Lynn Stothers, MD, MHSc, FRCSC ; Adrian Wagg, MD, FRCP (Lond), FRCP (Edin), FHEA ${ }^{6}$
}

'Department of Urology, Jewish General Hospital, McGill University, Montreal, QC; ${ }^{2}$ Northern Alberta Urology Centre, Edmonton, AB; ${ }^{3}$ Division of Urology, Department of Surgery, The Ottawa Hospital, Ottawa, ON; ${ }^{4}$ Division of Urology, Toronto Western Hospital, University of Toronto, University Health Network, Toronto, ON; ${ }^{5}$ Department of Urological Sciences, University of British Columbia, Vancouver, $\mathrm{BC} ;{ }^{6}$ Department of Medicine, University of Alberta, Edmonton, AB; Canada

Cite as: Can Urol Assoc J 2017:11(5):E248-9.

Pubished online May 9, 2017

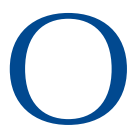
veractive bladder $(\mathrm{OAB})$ is a symptom syndrome consisting of urinary urgency with or without urge incontinence, often accompanied by frequency and nocturia, in the absence of urinary tract infection (UTI) or other obvious pathology. OAB is common in both sexes, with increasing prevalence with age. The overall prevalence of $\mathrm{OAB}$ in a Canadian population is estimated at $14-18 \%$. Most patients have a combination of OAB symptoms. In men with benign prostatic hyperplasic $(\mathrm{BPH}), \mathrm{OAB}$ and bladder outlet obstruction (BOO) often coexist (Evidence strength Grade $B$ ).

Since $O A B$ is not life-threatening, its impact on quality of life (QOL) plays a major role in the decision to treat patients. The significant negative impact that $O A B$ has on daily activities, mental health, sexual function, and marital satisfaction has been highlighted by a number of studies. Furthermore, $\mathrm{OAB}$ symptoms are linked to depressive illness. Usually, a distinction is made between QOL and well-being in those patients with incontinence $(\mathrm{OAB}$-wet) and those without (OAB-dry). Individuals who develop urinary incontinence (UI) have worse QOL. There is limited evidence concerning the psychosocial impact of $\mathrm{OAB}$ in either frail or multimorbid older persons; however, currently available data suggest that it is a serious concern.

Patients with $\mathrm{OAB}$ require comprehensive assessment. There is a universal agreement that taking a history should be the first step in the assessment of OAB patients (Evidence strength Grade $B$ ). Clinical examination should be part of assessment of people with OAB (Expert opinion).

Patient self-completed questionnaires are the most suitable method for assessing the patient's perspective of bothering symptoms and further implications on patients' QOL (Evidence strength Grade B). Questionnaires should have been validated for English or French. Measurement of the micturition frequency and fluid intake habits should be performed with voiding diaries (Evidence strength Grade B). It is recommended to perform a voiding diary observation of 3-7 days' duration.

As OAB symptoms may occur during symptomatic UTI, urinalysis should be included in the initial evaluation of all patients suspected of OAB (Evidence strength Grade C). Post-voiding residual (PVR) volume measurement, bladder/ renal ultrasound, cystoscopy, computed tomography (CT), magnetic resonance imaging (MRI), and urodynamic study (UDS) are not recommended in the initial diagnosis process of the uncomplicated OAB patients (Evidence strength $G$ rade $A / B / C$ ). Additional tests are indicated when the diagnosis remains uncertain after history and physical examination, when the symptoms do not correlate with physical findings, or after failed previous treatment (Expert opinion).

Behavioural therapies and lifestyle changes should be the first-line therapy in all patients because of the non-invasive nature of the treatment. Bladder training and pelvic floor muscle therapy may be effective methods of treatment in certain cases (Evidence strength Grade B). Lifestyle changes involving modifications of fluids/caffeine intake, weight control, dietary modifications, management of bowel regularity, and optimization of other comorbidities (i.e., diabetes, congestive heart failure, obstructive sleep apnea) can be effective (Evidence strength Grade B/C).

Patient education empowers patients and engages them in their treatment plan. First-line treatments for $O A B$ strongly rely on patient compliance and adherence. Patients with $\mathrm{OAB}$ and UI have an improved QOL when they have a comprehensive knowledge of their medical problem and seek to develop an avoidance-oriented, stress-coping lifestyle that promotes social contact. Knowing about the condition that causes their symptoms can aid in the adoption of measures that lower the severity and impact of the condition (Expert opinion). 
Second-line treatment of $O A B$ should include the use of oral antimuscarinics (AMs), transdermal oxybutynin or oral beta-3-adrenoceptor agonist (Evidence strength Grade A). The lowest recommended dose should first be prescribed, followed by increasing the dose to obtain the best clinical improvement while monitoring for adverse events (Evidence strength Grade B). If the initial selected drug is not tolerated or does not provide adequate symptom relief, patients should be offered an alternative medication, preferably with a different mechanism of action (Expert opinion). The adverse event profile and possible contraindications should be considered when prescribing the drug of choice as second-line treatment (Expert opinion). Immediate-release formulations of AMs should be avoided if other formulations are available (Evidence strength Grade A). Patients who remain incontinent after the initial treatment with an AM could be offered combination treatment with solifenacin and mirabegron (Evidence strength Grade C).

Age-related changes in pharmacokinetics affect AM drugs for $\mathrm{UI}$ and these factors should be incorporated into treatment planning (Evidence strength Grade $\boldsymbol{B}$ ). Drugs may be effective at lower doses in frailer compared with healthier older persons (Evidence strength Grade C). Polypharmacy increases the chance of adverse reactions to drug therapy, which are more common in the frail elderly (Evidence strength Grade A). Furthermore, drug-drug and drug-disease interactions are common in frail older persons (Evidence strength Grade $\boldsymbol{A} / \boldsymbol{B})$. AMs for treatment of $\mathrm{OAB}$ remain as potentially inappropriate medications for frail older people (Evidence strength Grade B/C). Cholinesterase inhibitor treatment is associated with either precipitation or worsening of OAB symptoms (Evidence Strength Grade C).
OnabotulinumtoxinA (100 U) may be offered as longterm therapy to carefully selected patients with symptoms of frequency, urgency, and urgency incontinence who have had an inadequate response to or are intolerant of $\mathrm{OAB}$ pharmacotherapy (Evidence strength Grade A). Patients considering onabotulinumtoxinA must be carefully counselled regarding the need for close followup, the possible need for catheterization (indwelling or clean intermittent catheterization), and likelihood of repeat injections to maintain symptom improvement.

Physicians and patients should consider peripheral tibial nerve stimulation as safe and effective as third-line treatment in a carefully selected population (Evidence strength Grade B). Sacral neuromodulation should be considered as more invasive and higher-risk than other third-line treatments, but a suitable option for patients with OAB symptoms refractory to preferred treatment options (Evidence strength Grade B). Indwelling catheterization, augmentation cystoplasty, or other urinary diversions are rare long-term management strategies for $\mathrm{OAB}$ and should only be taken into account after all other medical and surgical options have been exhausted and only after careful consideration of the likely benefits and risks (Evidence strength Grade $\boldsymbol{D}$ ).

Patient followup should be routinely offered and individualized based on current treatment(s) and concern for patient safety. At each followup visit, the clinician should assess for compliance, efficacy, and potential side effects. If management is deemed ineffective or intolerable, then alternative treatment options should be presented, including drug dose-modification, change within drug class, change or addition of drug class, or consideration of third- or, rarely, additional OAB therapies (Expert Opinion). 